\title{
An upper bound for the Lipschitz retraction constant in $l_{1}$
}

\author{
by \\ Marco Annoni and Emanuele Casini (Como)
}

\begin{abstract}
We construct a new lipschitzian retraction from the closed unit ball of the Banach space $l_{1}$ onto its boundary, with Lipschitz constant 8 .
\end{abstract}

1. Introduction and notation. Given a Banach space $(X,\|\cdot\|)$, let $B(X)=\{x \in X:\|x\| \leq 1\}$ denote the closed unit ball, and $S(X)=$ $\{x \in X:\|x\|=1\}$ the unit sphere.

We know, by Benyamini-Sternfeld's theorem (see [2]), that $X$ is infinitedimensional if and only if there exists a lipschitzian retraction from $B(X)$ onto $S(X)$, i.e., a lipschitzian function which fixes all points in $S(X)$. Let $\mathcal{R}_{X}(k)$ (resp. $\mathcal{L}_{X}(k)$ ) denote the set of $k$-lipschitzian retractions (resp. maps) from $B(X)$ onto $S(X)$ (resp. into $B(X)$ ). If $X$ is infinite-dimensional, we define the retraction constant of $X$ to be

$$
k_{0}(X)=\inf \left\{k \in \mathbb{R}^{+}: \mathcal{R}_{X}(k) \neq \emptyset\right\} .
$$

The problem to exactly determine $k_{0}(X)$ for at least one Banach space $X$ is still open. Some bounds are however known:

- $k_{0}(X) \geq 3$ for every Banach space (see [5]);

- $k_{0}(H) \geq 4.55$ for every Hilbert space $H$ (see [5]);

- $k_{0}\left(l_{1}\right) \geq 4$ (see [3]);

- $k_{0}(X) \leq 30.84$ for every Banach space $X$ in which $\psi_{X}(k)=1-1 / k$ (see [1]), where

$$
\psi_{X}(k)=\sup _{T \in \mathcal{L}_{X}(k)} \inf _{x \in B(X)}\|x-T x\|,
$$

in particular, $k_{0}\left(c_{0}\right) \leq 30.84$;

- $k_{0}(H) \leq 28.99$ (see $\left.[1]\right)$;

- $k_{0}(\mathcal{C}([0,1])) \leq 4(1+\sqrt{2})^{2} \simeq 23.31$ (see $\left.[4]\right)$;

- $k_{0}\left(l_{1}\right) \leq 22.45$ (see $\left.[1]\right)$;

2000 Mathematics Subject Classification: Primary 47H10.

Key words and phrases: retractions, retraction constant, Lipschitz maps. 
- $k_{0}\left(\mathcal{C}_{0}([0,1])\right) \leq 17.38$ (see $\left.[5]\right)$, where $\mathcal{C}_{0}([0,1]):=\{f \in \mathcal{C}([0,1])$ : $f(0)=0\}$ is endowed with its natural norm;

- $k_{0}\left(L_{1}([0,1])\right) \leq 9.43([5])$.

The last upper bound has been the best known so far for any Banach space. Here we improve this result by exhibiting an 8-lipschitzian retraction from $B\left(l_{1}\right)$ onto $S\left(l_{1}\right)$.

For further results about the retraction constant and the function $\psi_{X}$ we refer the reader to [5].

\section{The retraction in $l_{1}$. Let}

$$
B_{1}=\left\{x \in l_{1}:\|x\| \leq 1 / 2\right\}, \quad B_{2}=\left\{x \in l_{1}: 1 / 2 \leq\|x\|<1\right\},
$$

and define $i: B_{2} \rightarrow \mathbb{N}$ by setting, for $x=\left(x_{j}\right)_{j=0}^{\infty} \in l_{1}$,

$$
i(x)=\min \left\{j \in \mathbb{N}: \sum_{k=j+1}^{\infty}\left|x_{k}\right|<1-\|x\|\right\} .
$$

Moreover, let $\mu: B_{2} \rightarrow(0,1]$ be such that, for all $x \in B_{2}$,

$$
\mu(x)\left|x_{i(x)}\right|+\sum_{k=i(x)+1}^{\infty}\left|x_{k}\right|=1-\|x\|,
$$

and define $Q: \bar{B}_{2} \rightarrow B_{1}$ by

$$
Q(x)= \begin{cases}\mu(x) x_{i(x)} e_{i(x)}+\sum_{k=i(x)+1}^{\infty} x_{k} e_{k} & \text { if }\|x\|<1, \\ 0 & \text { if }\|x\|=1,\end{cases}
$$

where $\left\{e_{j}\right\}_{j=0}^{\infty}$ is the standard basis of $l_{1}$ and $\bar{B}_{2}$ is the closure of $B_{2}$. Observe that, for any $x \in B_{2}$, we have

$$
\|Q(x)\|=1-\|x\|
$$

and so

$$
\|(I-Q)(x)\|=\|x\|-\|Q(x)\|=2\|x\|-1,
$$

where $I$ is the identity map.

In the following proposition we give the main properties of the map $Q$.

Proposition 1. $Q$ is 3-lipschitzian and $I-Q$ is 2-lipschitzian.

Proof. If $\|x\|=1$ and $y \in \bar{B}_{2}$ then

$$
\begin{aligned}
\|(I-Q)(x)-(I-Q)(y)\| & \leq\|x-y\|+\|Q(x)-Q(y)\| \\
& =\|x-y\|+\|0-Q(y)\|=\|x-y\|+(1-\|y\|) \\
& =\|x-y\|+\|x\|-\|y\|=2\|x-y\| .
\end{aligned}
$$


Now suppose that $x, y \in B_{2}$. We will discuss only the case $i(x) \neq i(y)$ (the case $i(x)=i(y)$ is analogous and left to the reader). We can suppose that $i(x)<i(y)$. Assuming that $\sum_{k=n}^{m} a_{k}=0$ if $m<n$, we have

$$
\begin{aligned}
\| & (I-Q)(x)-(I-Q)(y) \| \\
= & \sum_{k=0}^{i(x)-1}\left|x_{k}-y_{k}\right|+\left|(1-\mu(x)) x_{i(x)}-y_{i(x)}\right|+\sum_{k=i(x)+1}^{i(y)-1}\left|y_{k}\right|+(1-\mu(y))\left|y_{i(y)}\right| \\
\leq & \sum_{k=0}^{i(x)}\left|x_{k}-y_{k}\right|+\mu(x)\left|x_{i(x)}\right|+\sum_{k=i(x)+1}^{i(y)}\left|y_{k}\right|-\mu(y)\left|y_{i(y)}\right| \\
= & \sum_{k=0}^{i(x)}\left|x_{k}-y_{k}\right|+\left(1-\sum_{k=0}^{\infty}\left|x_{k}\right|-\sum_{k=i(x)+1}^{\infty}\left|x_{k}\right|\right)+\sum_{k=i(x)+1}^{i(y)}\left|y_{k}\right| \\
& -\left(1-\sum_{k=0}^{\infty}\left|y_{k}\right|-\sum_{k=i(y)+1}^{\infty}\left|y_{k}\right|\right) \\
= & \sum_{k=0}^{i(x)}\left|x_{k}-y_{k}\right|+\sum_{k=0}^{\infty}\left(\left|y_{k}\right|-\left|x_{k}\right|\right)+\sum_{k=i(x)+1}^{\infty}\left(\left|y_{k}\right|-\left|x_{k}\right|\right) \leq 2\|x-y\| .
\end{aligned}
$$

So $I-Q$ is 2-lipschitzian. It follows that $Q$ is 3-lipschitzian (just write $Q=I-(I-Q))$.

Finally, if $\sigma$ denotes the "right-shift" map, we define $R: B\left(l_{1}\right) \rightarrow S\left(l_{1}\right)$ by

$$
R(x)= \begin{cases}(1-2\|x\|) e_{0}+2 \sigma(x) & \text { if } x \in B_{1}, \\ (I-Q)(x)+2 \sigma(Q(x)) & \text { if } x \in \bar{B}_{2},\end{cases}
$$

and let $R_{1}: \equiv R_{\mid B_{1}}$ and $R_{2}: \equiv R_{\mid \bar{B}_{2}}$. It can be easily verified that:

- $R$ is well defined;

- $R_{1}$ is 4 -lipschitzian and $R_{2}$ is 8 -lipschitzian;

- $R$ is lipschitzian with Lipschitz constant $\operatorname{lip}(R)=\max \left\{\operatorname{lip}\left(R_{1}\right), \operatorname{lip}\left(R_{2}\right)\right\}$ $\leq 8$

- $R(x) \in S\left(l_{1}\right)$ for all $x \in B\left(l_{1}\right)$;

- $R_{2}(x)=x$ if $\|x\|=1$, so that $R$ is a retraction onto $S\left(l_{1}\right)$.

This obviously shows that $k_{0}\left(l_{1}\right) \leq 8$.

\section{References}

[1] M. Baronti, E. Casini and C. Franchetti, The retraction constant in some Banach spaces, J. Approx. Theory 120 (2003), 296-308. 
[2] Y. Benyamini and Y. Sternfeld, Spheres in infinite dimensional normed spaces are Lipschitz contractible, Proc. Amer. Math. Soc. 88 (1983), 439-445.

[3] K. Bolibok, Minimal displacement and retraction problems in the space $l_{1}$, Nonlinear Anal. Forum 3 (1998), 13-23.

[4] K. Goebel, On the problem of retracting balls onto their boundary, Abstr. Appl. Anal. 2003, no. 2, 101-110.

[5] K. Goebel and W. A. Kirk, Topics in Metric Fixed Point Theory, Cambridge Univ. Press, 1990.

Marco Annoni

Via Salita Cappuccini 16

22100 Como, Italy

E-mail: marcoannoni.81@gmail.com
Emanuele Casini

Dipartimento di Fisica e Matematica

Università degli Studi dell'Insubria

Via Valleggio 11 22100 Como, Italy

E-mail: emanuele.casini@uninsubria.it

Received January 20, 2006

Revised version February 2, 2007 